\title{
Quality of Life Outcome Analysis of Robotic Radical Prostatectomy: A Single Center Experience
}

\author{
*Shashikant Mishra DNB Urology, Ankush Jairath Urology Resident, Jaspreet \\ chhabra, MCH Urology, Arvind Ganpule DNB Urology, Abhishek Singh MCH Urology, \\ Ravindra Sabnis MCH Urology, Mahesh Desai F.R.C.S. (Lond.) \\ Muljhibhai Patel Urological Hospital, NADIAD, INDIA \\ *mishra@mpuh.org
}

\begin{abstract}
:
Introduction: Robot assisted radical prostatectomy (RARP) is the standard of care treatment for localized prostate cancer. We report the quality of life $(Q o L)$ outcome in our initial 100 cases of RARP and determine factors affecting outcome.

Materials and Methods: We evaluated five parameters; baseline and postoperative validated Hindi and English version questionnaires (International Index of Erectile Function-5, NationalCancerInstitute-2 continence grading, Functional Assessment of Cancer Therapy-Prostate [FACT-P] scores) for erectile, continence and QoL outcome, peri-operative morbidity and oncological success in 100 patients undergoing $R A R P$.

Results: The average age of the patients was $63.6 \pm 6.6$ years. $p T 2 a, p T 2 b, p T 2 c$ and pT3 tumors were $16.5 \%$, $7.7 \%, 36.3 \%$ and $39.5 \%$, respectively. The hemoglobin drop was $1.63 \pm 0.48 \mathrm{mg} / \mathrm{dl}$. Complications were noted in 16.5\%, majority being Clavien 1 (15.4\%). Surgical margin was positive in $13.2 \%$ patients. Average follow-up duration was $15.9 \pm 7.4$ months. Adjuvant and salvage radiotherapy was given in 26 and 5 patients, respectively. 2-year cancer specific survival was 100\%. Moderate erectile dysfunction was present in 59.4\%, $98.4 \%$, and $86.2 \%$ at baseline, 12 and 24 months, respectively. Continence at 3, 6, and 12 months was 77\%, $81 \%$ and $92 \%$ respectively. At 6 months follow-up, most patients had recovered FACT-P scores. Age (0.03), Charlson co-morbidity index (0.04) and pathological stage (0.02) affected FACT-P scores.
\end{abstract}

Conclusion: Robot assisted radical prostatectomy is associated with minimal morbidity and good oncological outcome. Most QoL indices recover within 6 months. Our patient cohort reported poor erectile outcome.

Keywords: Erectile function, oncological outcome, quality of life, robotic assisted radical prostatectomy

\section{INTRODUCTION}

Radical prostatectomy (RP) has become the most widespread treatment for clinically localized cancer prostate $(\mathrm{CaP}) .{ }^{[1]} \mathrm{RP}$ has been evaluated with the trifecta results aiming to measure oncological outcome, potency, and continence. ${ }^{[2]}$ The functional outcome corresponds to the overall health related quality of life (HRQoL) and its assessment is important for counseling the patients for surgery. With the introduction of minimally invasive surgery like laparoscopy or robotics, there have been significant advantages of early recovery. Robotic assisted radical prostatectomy (RARP) is increasingly utilized in the United States and is the most common robotic procedure performed worldwide. ${ }^{[3-5]}$ Given the advantage of excellent visualization and ability to achieve meticulous nerve dissection, faster urinary and sexual function recovery in RARP patients have been reported. ${ }^{[6]}$ Collectively, they improve the so- called pentafecta results and contribute to early return of preoperative HRQoL. ${ }^{[7]}$

In this study, we assessed quality of life (QoL) outcomes analyzing oncological outcome, perioperative morbidity and variables affecting HRQoL, continence and potency outcome after RARP.

\section{Materials AND Methods}

This is a prospective single center, non-comparative study. All patients with clinically localized CaP who underwent RARP at our institution between September 2011 and March 2013 (first hundred patients) were studied. Prostate specific antigen (PSA), contrast enhanced computed tomography scan of abdomen and whole body $99 \mathrm{mTc}$-bone scan were done as a part of staging $\mathrm{CaP}$ in appropriate patients. Preoperative demographic data including age, body mass index (BMI), education, 
occupation, socioeconomic status, Charlson co morbidity index (CCI), international prostatic symptom score, PSA, clinical tumor, node, metastases stage and biopsy Gleason grade and score were recorded. This study included a list of theoretically pertinent demographic criterion, such as the socioeconomic status (according to Kuppuswamy's socioeconomic status scale), ${ }^{[8]}$ education (illiterate, primary, secondary, and higher education), comorbidities (diabetes, hypertension, ischemic heart disease, obstructive lung disease, and chronic kidney disease), present occupation (retired sedentary, retired active life, and professional) and residence (urban and rural).

Patients were stratified in the following risk groups; low-risk: PSA $10 \mathrm{ng} / \mathrm{mL}$ and a Gleason score of 6 or less and clinical stage T1c or T2a; intermediate risk: PSA $>10-20 \mathrm{ng} / \mathrm{mL}$ or a Gleason score of 7 or clinical stage T2b, but not qualifying for high-risk; high-risk: PSA $>20 \mathrm{ng} / \mathrm{mL}$ or a Gleason score of 8-10 or clinical stage T2c. All clinically localized CaP patients not willing for active surveillance and radiotherapy were explained regarding RP. RARP was performed by standard technique. Decision to preserve or excise neuro-vascular bundle was taken by the operating surgeon considering the local $\mathrm{CaP}$ staging. Mostly, patient with high-risk localized $\mathrm{CaP}$ was not considered for neuro-vascular bundle preservation. A note was made for operative duration, estimated blood loss, nerve sparing and any intraoperative complication. Patients were kept in the hospital till the postoperative cystogram was performed at day 5. In the absence of extravasation, the urethral catheter was removed. Patients were further observed for 1 day and then discharged. In the presence of extravasation, the catheter was placed for a further period of 1 week. In that scenario, the patient was discharged and called back for catheter removal on a specified date. Peri-operative morbidity was defined as morbidity occurring within 30 days after the operation. Hospital stay, catheter duration and peri-operative morbidity were also recorded for each patient. In all the patients scheduled for RP, a designated physiotherapist started pelvic exercise at the first outpatient visit. It was continued during the preoperative period. Postsurgery, it was restarted as and when the patient felt comfortable; usually on $2^{\text {nd }}$ postoperative day. It was continued until the patient gained continence. This was done in all the patients. No penile rehabilitation protocol was followed in this cohort of patients. Patients were encouraged during postoperative period to continue waiting for the return of erectile function without the need for active intervention. ${ }^{[9]}$

Prostate specific antigen was assessed at 3 monthly intervals. Biochemical recurrence (BCR) was defined as two consecutive PSA level $>0.4 \mathrm{ng} / \mathrm{mL}$ after RARP. ${ }^{[10]}$ In appropriate patients, adjuvant radiotherapy was offered as early as 3 months postoperatively or else salvage radiotherapy was advised. Hormonal treatment was offered in 3 patients of node positive disease. Postoperative surgical margin positivity, PSA BCR and need for adjuvant treatment after RARP were used as surrogate for oncological outcome. Preoperative baseline assessment of continence, erectile function and HRQoL was done in all patients. Postoperative assessment of continence and erection was done at 1, 3, 6 and 12 months, while HRQoL assessment was done at 3 months.

Urinary incontinence (UI) was evaluated using a patient self-assessed questionnaire, based on the National Cancer Institute (NCI) Common Toxicity Criteria (version 2). Grade 0 was defined as no incontinence; Grade 1 as incontinence occurring with coughing, sneezing, or laughing; Grade 2 as spontaneous incontinence with some control; and Grade 3 as no control of urine in the absence of any fistula. ${ }^{[1]}$ Postoperative continence was defined when patient had either Grade 0 or Grade 1 continence. ${ }^{[12]}$

Erectile dysfunction (ED) was defined as the persistent inability to achieve and maintain an erection sufficient to permit satisfactory sexual intercourse with or without use of phosphodiesterase (PDE) inhibitor, intracavernosal injection or vacuum erection device. ${ }^{[13-15]}$ Erectile function was assessed using English or validated Hindi version of "abridged five-item of the International Index of Erectile Function (IIEF-5)" questionnaire. ${ }^{[16]}$ This questionnaire consists of five questions and each IIEF-5 item is scored on a five-point ordinal scale measuring five domains of sexual function namely; erectile and orgasmic functions, sexual desire, satisfaction with intercourse and overall sexual satisfaction. The possible scores for the IIEF-5 range from 5 to 25 . ED was classified into five categories based on the scores: Severe (5-7), moderate (8-11) mild to moderate(12-16), mild(17-21), and no ED(2225). ${ }^{[16,17]}$

We used Functional Assessment of Cancer Therapy-Prostate (FACT-P) scores that provide useful summary of overall HRQoL ${ }^{[6]}$. FACT-P is a reliable and scientifically valid, patient reported survey 
for $\mathrm{CaP}$ patients to evaluate five independent subscales measuring physical well-being (PWB-7 items, score range: 0-28), family/social well-being (SWB-7 items, score range: 0-28), emotional well-being (EWB-6 items, score range: 0-24), functional well-being (FWB-7 items, score range: 0-28) and prostate cancer subscale (PCS-12 items, score range: $0-48$ ). The FACT-P total score is calculated by adding these five subscales and ranges from 0 to 156. A higher score indicates better HRQoL. Severe worsening of postoperative FACT-P score was defined as decrease from the individual baseline score of more than one standard deviation (SD), significant improvement in postoperative scores was defined using the same one SD cutoff.

The social work department (MSW) assistant administered the FACT-P and IIEF-5 questionnaires in an interview format. All the questionnaires were given in either English or validated Hindi language data sheet that the patients were required to fill up either alone or in the presence of their family members. Prior to the start of the study, adequate training of the interviewers was done to elicit nonbiased responses. Patients were encouraged to enter every single item without skipping any. The FACT-P score was considered to be an acceptable indicator of patient's HRQoL as long as overall item response rate was $>80 \%$. The subscale item response was prorated for missing items if $>50 \%$ of items were answered. The validated Hindi version FACT-P and IIEF-5 was scored on English language scoring guides.

A single researcher performed the objective scoring of the three functional outcome parameters. ${ }^{[6]}$ Multivariate conditional logistic regression analysis with forward selection was performed of the possible strong factors from univariate analysis to determine adjusted odds ratio (ORs). $P<0.05$ was considered as statistically significant.

\section{RESULTS}

In all, 100 patients underwent RARP during the study period. Minimum 3 months follow up data was available in 94 patients. Three patients died with unrelated causes and were excluded from the analysis. The full HRQoL data were available in 91 patients. The demographic profile of the patients is as in Table 1. The average age of the patients was $63.6 \pm 6.6$ years. Twenty-seven patients had screen detected CaP. The preoperative PSA was $18.0 \pm 16.4 \mathrm{ng} / \mathrm{mL}$.

Table1. Demography

\begin{tabular}{|c|c|}
\hline Variables & $\mathbf{n}$ \\
\hline Number of cases & 91 cases \\
\hline Age (years); mean \pm SD & $63.6 \pm 6.6$ \\
\hline$\leq 55$ & 14 \\
\hline $55-65$ & 39 \\
\hline$\geq 65$ & 38 \\
\hline BMI $\left(\mathrm{kg} / \mathrm{m}^{2}\right) ;$ mean \pm SD & $26.1 \pm 2.42$ \\
\hline \multicolumn{2}{|l|}{$\mathrm{CCI}$} \\
\hline 0 & 51 \\
\hline 1 & 29 \\
\hline 2 & 11 \\
\hline \multicolumn{2}{|l|}{ CaP detection $(n)$} \\
\hline Screening & 27 \\
\hline LUTS & 60 \\
\hline Post TURP & 4 \\
\hline Preoperative PSA $(\mathrm{ng} / \mathrm{mL}) ;$ mean $\pm \mathrm{SD}$ & $18.01 \pm 16.38$ \\
\hline$<4$ & 4 \\
\hline$\geq 4-<10$ & 28 \\
\hline$\geq 10-<20$ & 34 \\
\hline$\geq 20-<50$ & 18 \\
\hline$\geq 50$ & 7 \\
\hline \multicolumn{2}{|c|}{ Preoperative clinical stage $(n)$ with Gleason score $(6,7, \geq 8)$} \\
\hline T1c & $15(9,4,2)$ \\
\hline $\mathrm{T} 2 \mathrm{a}$ & $22(17,3,2)$ \\
\hline $\mathrm{T} 2 \mathrm{~b}$ & $19(9,7,3)$ \\
\hline $\mathrm{T} 2 \mathrm{c}$ & $35(14,15,6)$ \\
\hline Histopathology stage; Gleason $(6,7, \geq 8)$ & \\
\hline
\end{tabular}


Shashikant Mishra et al.

\begin{tabular}{|l|l|}
\hline \hline pT2aN0 & $15(10,3,2)$ \\
\hline pT2bN0 & $7(1,5,1)$ \\
\hline pT2cN0 & $32(10,19,3)$ \\
\hline pT2cN1 & $1(0,1,0)$ \\
\hline pT3aN0 & $19(1,16,2)$ \\
\hline pT3bN0 & $14(1,7,6)$ \\
\hline pT3bN1 & $3(0,1,2)$ \\
\hline Baseline incontinence score (NCI Common Toxicity Criteria [version 2]) & \\
\hline Grade 0 & 91 \\
\hline Baseline IIEF-5 score (mean \pm SD) & $14.15 \pm 7.06$ \\
\hline Baseline FACT-P score, range: 0-156 (mean+SD) & $120.06 \pm 10.25$ \\
\hline PWB, range: 0-28 (mean \pm SD) & $17.75 \pm 2.9$ \\
\hline SWB, range: 0-28 (mean \pm SD) & $19.8 \pm 3.44$ \\
\hline EWB, range: 0-24 (mean \pm SD) & $19.02 \pm 2.60$ \\
\hline FWB, range: 0-28 (mean \pm SD) & $19.24 \pm 2.19$ \\
\hline PCS, range: 0-48 (mean \pm SD) & $44.24 \pm 1.30$ \\
\hline
\end{tabular}

$S D=$ Standard deviation, BMI=Body mass index, CCI=Charlson co morbidity index, CaP=Cancer prostate, LUTS=Lower urinary tract symptoms, TURP=Transurethral resection of the prostate, PSA=Prostate specific antigen, IIEF=International Index of Erectile Function, $P W B=$ Physical well-being, SWB=Social well-being, EWB=Emotional well-being, $F W B=$ Functional well-being, PCS=Prostate cancer subscale, FACT$P=$ Functional Assessment of Cancer Therapy-Prostate, NCI=National Cancer Institute

Unilateral and bilateral neuro-vascular preservation was done in 55 and 7 patients, respectively. The total operative time was $183.1 \pm 47.6 \mathrm{~min}$. Three patients required one blood transfusion each. The hemoglobin drop was $1.63 \pm 0.48 \mathrm{mg} / \mathrm{dl}$. Complications were noted in $16.5 \%$, majority being Clavien 1 (15.4\%). Postoperative Clavien 1 complications noted were urine leak (4), epidydmo-orchitis (1), lymphocele (1), retention (5) and paralytic ileus (3), while 1 patient had Clavien 3 complication (bleeding) requiring open conversion. The mean catheter duration and hospital stay were $5.2 \pm 2.3$ and $7.8 \pm 1.9$ days, respectively. Pathological stage with Gleason $6,7, \geq 8$ is given in Table 1. Surgical margin was positive in $13.2 \%$ patients. Average follow up duration was $15.9 \pm 7.4$ months. Of the 91 evaluable patients, 91, 83, 66, and 31 patients had completed 3, 6, 12, and 24 months follow-up visit after RARP. Interim 2-year cancer specific survival was $100 \%$. Three patients died due to unrelated diseases. PSA recurrence free survival for pT2N0 and pT3, N0-1 was 92.6\% and 86.5\%, respectively. Adjuvant and salvage radiotherapy was given in 26 and 5 patients, respectively.

Based on the self-administered questionnaire, $59.4 \%$ patients at baseline had more or equal to mild to moderate ED. At 12 and 24 months follow-up, $98.4 \%$ and $86.2 \%$ still persisted to have more or equal to mild to moderate ED, respectively. Of the initial data set of $18(20 \%)$ patients who were potent (score >20), none had recovery of potency when followed until 12 months. At 12 months follow-up, there were $6(30 \%), 8(40 \%), 4(20 \%)$ and $2(10 \%)$ patients who had scores in the severe, moderate, mild to moderate and mild ED range.

Continence data of the patients is given in Table 2. All patients were continent prior to RARP. At 1, 3, 6,12 , and 24 months follow-up, $41 \%, 77 \%, 81 \%, 92 \%$, and $91 \%$ patients respectively, were continent (Grades 0 and 1).

Table2. Continence Outcome

\begin{tabular}{|l|l|l|l|l|l|l|}
\hline Continence & $\begin{array}{c}\text { Baseline } \\
\boldsymbol{n}(\boldsymbol{\%})\end{array}$ & $\begin{array}{c}\text { 1 month } \boldsymbol{n} \\
(\boldsymbol{\%})\end{array}$ & $\begin{array}{c}\text { 3 months } \boldsymbol{n} \\
(\mathbf{\%})\end{array}$ & $\begin{array}{c}\text { 6 months } \boldsymbol{n} \\
(\boldsymbol{\%})\end{array}$ & $\begin{array}{c}\mathbf{1 2} \text { months } \boldsymbol{2} \\
(\boldsymbol{\%})\end{array}$ & $\begin{array}{c}\text { 24 months } \boldsymbol{n} \\
(\boldsymbol{\%})\end{array}$ \\
\hline Grade 0 & $91(100)$ & $7(7.7)$ & $28(30.8)$ & $52(62.5)$ & $46(70.8)$ & $23(71.9)$ \\
\hline Grade 1 & $0(0.0)$ & $30(32.9)$ & $42(46.1)$ & $15(18.1)$ & $14(21.5)$ & $6(18.7)$ \\
\hline Grade 2 & $0(0.0)$ & $51(56.1)$ & $20(21.9)$ & $11(13.2)$ & $5(7.7)$ & $2(6.2)$ \\
\hline Grade 3 & $0(0.0)$ & $3(3.3)$ & $1(1.1)$ & $1(1.2)$ & $0(0.0)$ & $0(0.0)$ \\
\hline
\end{tabular}

Functional Assessment of Cancer Therapy-Prostate scores of PWB, SWB, EWB, FWB and PCS at baseline visit and postoperative follow-up at one, 3, 6, 12 and 24 months are as shown in Table 3. At 6 months follow up; most patients had recovered HRQoL. 
Quality of Life Outcome Analysis of Robotic Radical Prostatectomy: A Single Center Experience

Table3. FACT_P Outcome

\begin{tabular}{|l|l|l|l|l|l|l|}
\hline FACT-P & \multicolumn{1}{|c|}{ Baseline } & \multicolumn{1}{|c|}{ 1 month } & \multicolumn{1}{|c|}{ months } & 6 months & 12 months & 24 months \\
\hline PWB & $17.7 \pm 2.9$ & $14.5 \pm 2.5$ & $17.3 \pm 2.9$ & $17.9 \pm 2.9$ & $18.3 \pm 3.4$ & $18.7 \pm 3.7$ \\
\hline SWB & $19.8 \pm 3.4$ & $16.1 \pm 3.4$ & $14.7 \pm 3.1$ & $17.1 \pm 2.8$ & $17.3 \pm 3.1$ & $17.9 \pm 3.6$ \\
\hline EWB & $19.0 \pm 2.7$ & $15.9 \pm 2.3$ & $18.7 \pm 2.6$ & $19.2 \pm 2.6$ & $19.7 \pm 2.8$ & $19.4 \pm 0.4$ \\
\hline FWB & $19.2 \pm 2.2$ & $15.9 \pm 2.4$ & $18.2 \pm 2.7$ & $19.5 \pm 2.4$ & $19.7 \pm 2.4$ & $19.3 \pm 3.1$ \\
\hline PCS & $44.2 \pm 1.3$ & $42.5 \pm 1.3$ & $42.9 \pm 1.00$ & $43.3 \pm 1.0$ & $44.0 \pm 1.0$ & $44.6 \pm 1.1$ \\
\hline FACT-P (total) & $120.1 \pm 10.2$ & $105.0 \pm 9.5$ & $111.8 \pm 9.5$ & $117.1 \pm 9.6$ & $119.1 \pm 10.4$ & $120 \pm 12.7$ \\
\hline
\end{tabular}

$P W B=$ Physical well-being, $S W B=$ Social well-being, $E W B=$ Emotional well-being, $F W B=$ Functional well-being, $P C S=$ Prostate cancer subscale, FACT-P=Functional Assessment of Cancer Therapy-Prostate

The difference of FACT-P scores at 1 and 3 months was $15.2 \pm 3.1(0.036)$ and $8.3 \pm 3(0.064)$, respectively. We found that age, CCI, residence and pathological stage as the factors affecting HRQoL outcome with respect to FACT-P scores. The OR of these factors with 95\% confidence interval is mentioned in Table 4 . Of the 12 patients who had margin positivity and subsequently undergoing adjuvant radiotherapy, FACT-P score was statistically lower at 3 months $(P=0.03)$ but insignificant at 6 months $(P=0.68)$. At 3 months, most subscales including PWB, SWB, EWB, and PCS were significantly lower.

Table4. Adjusted Odds Ratio for QoL Outcome

\begin{tabular}{|l|l|l|l|}
\hline \multicolumn{1}{|c|}{ Variate } & \multicolumn{1}{c|}{$\boldsymbol{P}$ value } & \multicolumn{1}{c|}{ OR } & \multicolumn{1}{c|}{ 95\% CI } \\
\hline Age & 0.0375 & 2.0979 & $1.0438-4.2168$ \\
\hline CCI & 0.0426 & 2.0552 & $0.8795-4.8027$ \\
\hline Residence & 0.0320 & 0.1931 & $0.0368-1.0146$ \\
\hline P stage & 0.0210 & 0.6082 & $0.3987-0.9277$ \\
\hline OR=Odds ratio, CI=Confidence interval, CCI=Charlson co morbidity index \\
\hline
\end{tabular}

With respect to continence outcome, age ( $P$ 0.04, OR: 2.87) and pathological stage ( $P$ 0.037, OR: 1.5$)$ were the most important variable on multivariate regression at 1 and 3 months, respectively.

\section{DisCuSSION}

Cancer prostate accounts for nearly $33 \%$ of all newly diagnosed cancers in male. ${ }^{[18]}$ The reported incidence of adenocarcinoma prostate is low in India $(6 / 100,000)$ in comparison to USA $(200 / 100,000) .{ }^{[18]}$ Widespread PSA screening has led to more diagnosis of clinically localized CaP in young and healthier men, resulting in more longevity after the treatment. ${ }^{[19]}$ Therefore, there is a need for more critical evaluation of HRQoL outcome of different treatment modalities used for its treatment. The ideal treatment would be one that is oncologically safe and has minimal impact on HRQoL. With the acceptance of the minimally invasive treatment of $\mathrm{CaP}$, there is an increasing acknowledgement of the importance of maintaining the HRQoL. ${ }^{[2]}$ Since its introduction into the United States in 2000, RARP has widely grown in popularity. ${ }^{[20]}$ Contemporary comparison of published series is difficult because of differences in patient populations and methods of outcome assessment. ${ }^{[21,22]}$ We report single center Indian QoL outcomes after RARP done for localized CaP early in the learning curve.

The major impact on QoL is possibly due to UI and ED. Though most of the studies report that UI is usually self-limiting, ED may be prolonged. ${ }^{[23]}$ We observed that most patients when quantified objectively with the FACT-P scores of the HRQoL, returned to the baseline at $3^{\text {rd }}$ postoperative month follow-up. Since most patients were continent at follow-up, this suggests that the impact of ED was not much in their general well-being. Potency after RP is usually defined as the ability to maintain erections sufficiently rigid for penetration and sexual intercourse with or without the help of a PDE-5 inhibitor. ${ }^{[13-15]}$ The return of erectile function after RP correlates with the age of the patient, preoperative potency status and extent of nerve-sparing surgery. ${ }^{[24]} \mathrm{We}$ evaluated the IIEF-5 score at the baseline and interestingly, 59.4\% patients had more or equal to mild to moderate ED. In general, potency outcomes were poor; in addition to poor baseline potency. The impact of ED was reflected in the PCS domain of FACT-P HRQoL. In multivariate regression model, baseline erectile function score and age correlated with the outcome. Higher baseline score and younger age patients were more likely to report decreasing scores in PCS subscale of FACT-P. Most series reporting good potency outcomes are from RARP done for low/intermediate risk CaP. ${ }^{[22]}$ Screen detected cancer in our series were only $29.6 \%$. Wide resection and neuro-vascular bundle excision was done in $29(31.8 \%)$ cases and pathological non-organ confined cases were 37 (40.6\%). To summarize, we encountered more 
cases on the other end of the localized $\mathrm{CaP}$ spectrum. In such a scenario, preserving neuro-vascular bundle could compromise on positive margins. In addition, requirement of adjuvant radiation would negate all the perceived advantages of early erectile recovery.

The issue of continence is complex with single center, single institution physician studies ${ }^{[25,26]}$ reporting results that are markedly different from multi-institutional questionnaire based studies. ${ }^{[27]}$ There is a need to have an acceptable standard of measuring UI for better comparison among the contemporary series. ${ }^{[28]}$ The grading of pad free continence in the prostate cancer outcome study where $60.5 \%$ of the subjects reported pad free status when only $31 \%$ were actually truly continent underlines the importance of a lack in standardized evaluation. ${ }^{[28,29]}$ In order to provide a more subjective and objective assessment to a single center study, we chose to evaluate the issue of UI based on subject-based questionnaire. The least complicated that we could find was NCI criteria currently utilized in post radiation settings ${ }^{[30]}$ and infrequently even in post RP settings. ${ }^{[1,12]} \mathrm{We}$ hypothesized that it would offer the related measure outcome in an accurate manner.

We found that age was significant variable affecting continence status at $1^{\text {st }}$ postoperative month. Probably, younger patients were more motivated and performed Kegel's exercises actively. At $3^{\text {rd }}$ postoperative month, pathological stage was the most important variable affecting continence. In our series, majority of patients were high-risk localized $\mathrm{CaP} ; 26$ of them required adjuvant radiotherapy, still $81 \%$ and $92 \%$ were continent (Grade 0 and 1, respectively) by 6 and 12 months, respectively. Good continence data in the event of initial learning curve indicates indirect evidence in favor of robotics. Ficarra et al. ${ }^{[22]}$ in the meta-analysis found lesser UI with RARP when compared to open RP.

At 6 months follow-up; most of the patients had recovered HRQoL. For PWB, the relevant questions answered were lack of energy, feeling ill and retirement to bed. We found that higher age and lower socioeconomic status affected the PWB domain. For SWB, questions such as closeness to friends and partner and support from family and friends were asked. Young patients and continence status determined this domain outcome. We hypothesize that stigma of incontinence, especially in Indian family settings accounted for the negative response by the patient. EWB domain consisted of feeling sad and nervous, losing hope, worrying about the disease process and future. Younger age and active working life blunted the feeling of despair after the surgery. FWB was the ability to do the work, enjoy life, accept illness, and content feeling. The PCS consisted of ability to feel like man, difficulty in urinating and erectile ability. In both these domains, age was inversely related to higher score. This may explain more positive acceptance of the disease process in young age and vice versa. In terms of overall HRQoL, age, residence, CCI and pathological stage were found to statistically significant on multivariate logistic regression. Tseng et al., in their HRQoL assessment from an initial cohort of patients undergoing RARP reported that $19.2 \%, 76.8 \%, 98.7 \%$ and $90.6 \%$ of patients return to their baseline sexual, urinary, bowel and vitality domains at 1 -year, respectively. ${ }^{[31]}$ We found that most functional aspects (FACT-P scores) of HRQoL return to baseline by 3 months. The continence plateaus by 6 months, while potency outcomes cannot be commented. Shikanov et al ${ }^{[6]}$ used UCLAprostate cancer index SF-36 v2 questionnaire and found that the risk of a significant decrease in urinary function, urinary bother, sexual function and sexual bother was higher in patients with better baseline scores. Hollenbeck et al. found that an improvement in sexual health outcome was noted in a high volume urological practice. ${ }^{[32]}$ Berge et al. studied HRQoL prospectively and concluded that better urinary function was associated with better general mental health. ${ }^{[33]} \mathrm{A}$ similar study from China also found that age was significantly associated with several HRQoL domains. ${ }^{[34]}$ We also conclude that most HRQoL domains are affected by age. Older patients were more likely to have declined FACT-P scores. Better socioeconomic status results in better PWB domain. UI deteriorates family/SWB domain in older patients. Active working life improves EWB in younger patients.

We found that peri-operative morbidity of RARP cohort was non-inferior to our reported laparoscopic series. ${ }^{[35]}$ Specifically, catheter duration and hospital stay was short when compared to our laparoscopic series. ${ }^{[35]}$ Pasadena consensus panel in their meta-analysis, found that RARP is associated with less blood loss and transfusion rates compared with open RP. ${ }^{[36]}$ Most of our complications were Clavien 1. One patient required conversion to open to control bleeding from the right common iliac artery. This happened due to inadvertant penetrating injury from the right hand scissor instrument insertion by the assistant.

A rising serum PSA level is usually the earliest evidence of tumor recurrence. Surgical margin status and BCR have generally been used as surrogates for oncologic efficacy following RP. In most series 
of RARP, positive margin percentages decrease with experience. Pathologic tumor margins status seems to be comparable between laparoscopic, robotic, and open series overall. ${ }^{[22]}$ Our series is that of an early learning curve and consist primarily aggressive localized $\mathrm{CaP}$ patients. However, the margins status is still comparable to those reports by low volume single center. With more experience, the margins rates are assumed to decrease further.

\section{Conclusion}

Most patients in our series do not meet pentafecta outcome due to poor erectile outcomes. Therefore, pentafecta rate does not seem to be a logical outcome for our patients. In general, the outcomes depend on the patient population, tumor stage and treatment modality. Since there is a lack of widespread screening and overall lower incidence of $\mathrm{CaP}$, we need a more critical evaluation of the treatment outcome after RARP for better patient counseling. Unlike the West, we encounter more high-risk localized $\mathrm{CaP}$ where need for adjuvant treatment may alter the pentafecta outcome. As the experience grows, more mature data would be available. Until that time, more multicenter data is required to understand this aspect of treatment.

\section{REFERENCES}

[1] Walsh PC. Anatomic radical prostatectomy: Evolution of the surgical technique. J Urol 1998; 160:2418-24.

[2] Patel VR, Coelho RF, Chauhan S, Orvieto MA, Palmer KJ, Rocco B, et al. Continence, potency and oncological outcomes after robotic-assisted radical prostatectomy: Early trifecta results of a high-volume surgeon. BJU Int 2010; 106:696-702.

[3] Kaul S, Menon M. Robotic radical prostatectomy: Evolution from conventional to VIP. World J Urol 2006; 24:152-60.

[4] Miller J, Smith A, Kouba E, Wallen E, Pruthi RS. Prospective evaluation of short-term impact and recovery of health related quality of life in men undergoing robotic assisted laparoscopic radical prostatectomy versus open radical prostatectomy. J Urol 2007; 178:854-8; discussion 859.

[5] Dasgupta P, Kirby RS. Outcomes of robotic assisted radical prostatectomy. Int J Urol 2009; 16:244-8.

[6] Shikanov SA, Eng MK, Bernstein AJ, Katz M, Zagaja GP, Shalhav AL, et al. Urinary and sexual quality of life 1 year following robotic assisted laparoscopic radical prostatectomy. J Urol 2008; 180:663-7.

[7] Patel VR, Sivaraman A, Coelho RF, Chauhan S, Palmer KJ, Orvieto MA, et al. Pentafecta: A new concept for reporting outcomes of robot-assisted laparoscopic radical prostatectomy. Eur Urol 2011;59:702-7.

[8] Kumar BR, Dudala SR, Rao AR. Kuppuswamy's socioeconomic status scale-a revision of economic parameter for 2012. Int J Res Dev Health 2013;1:2-4.

[9] Hayat MJ, Howlader N, Reichman ME, Edwards BK. Cancer statistics, trends, and multiple primary cancer analyses from the surveillance, epidemiology, and end results (SEER) program. Oncologist 2007; 12:20-37.

[10] Heidenreich A, Aus G, Bolla M, Joniau S, Matveev VB, Schmid HP, et al. EAU guidelines on prostate cancer. Eur Urol 2008; 53:68-80.

[11] Bastide C, Kuefer R, Loeffler M, de Petriconi R, Gschwend J, Hautmann R. The role of radical prostatectomy in patients with clinically localized prostate cancer and a prostate-specific antigen level \& gt; 20 ng/ml. Prostate Cancer Prostatic Dis 2006; 9:239-44.

[12] Koehler N, Gansera L, Stolzenburg JU, Rebmann U, Truss MC, Roth S, et al. Early continence in patients with localized prostate cancer. A comparison between open retropubic (RRPE) and endoscopic extraperitoneal radical prostatectomy (EERPE). Urol Oncol 2012; 30:798-803.

[13] NIH Consensus Conference. Impotence. NIH Consensus Development Panel on Impotence. JAMA 1993; 270:83-90.

[14] Feldman HA, Goldstein I, Hatzichristou DG, Krane RJ, McKinlay JB. Impotence and its medical and psychosocial correlates: Results of the Massachusetts Male Aging Study. J Urol 1994; 151:54-61.

[15] Levine LA. Diagnosis and treatment of erectile dysfunction. Am J Med 2000; 109 Suppl 9A:3S12 ? 
[16] Rosen RC, Cappelleri JC, Smith MD, Lipsky J, Peña BM. Development and evaluation of an abridged, 5-item version of the International index of erectile function (IIEF-5) as a diagnostic tool for erectile dysfunction. Int J Impot Res 1999; 11:319-26.

[17] Cappelleri JC, Rosen RC. Reply to 'The sexual health inventory for men (IIEF-5)' by JA Vroege. Int J Impot Res 1999; 11:353-4.

[18] Morgans AK, Smith MR. Bone-targeted agents: Preventing skeletal complications in prostate cancer. Urol Clin North Am 2012;39:533-46.

[19] Bill-Axelson A, Holmberg L, Ruutu M, Häggman M, Andersson SO, Bratell S, et al. Radical prostatectomy versus watchful waiting in early prostate cancer. N Engl J Med 2005;352:1977-84.

[20] Altamar HO, Herrell SD. The current status of robot-assisted laparoscopic prostatectomy. Curr Opin Urol 2010;20:56-9.

[21] Berge V, Berg RE, Hoff JR, Wessel N, Diep LM, Karlsen SJ, et al. A prospective study of transition from laparoscopic to robot-assisted radical prostatectomy: Quality of life outcomes after 36-month follow-up. Urology 2013; 81:781-6.

[22] Ficarra V, Novara G, Fracalanza S, D'Elia C, Secco S, Iafrate M, et al. A prospective, nonrandomized trial comparing robot-assisted laparoscopic and retropubic radical prostatectomy in one European institution. BJU Int 2009; 104:534-9.

[23] Glickman L, Godoy G, Lepor H. Changes in continence and erectile function between 2 and 4 years after radical prostatectomy. J Urol 2009; 181:731-5.

[24] Walsh PC, Donker PJ. Impotence following radical prostatectomy: Insight into etiology and prevention. J Urol 1982; 128:492-7.

[25] Touijer K, Guillonneau B. Laparoscopic radical prostatectomy: A critical analysis of surgical quality. Eur Urol 2006; 49:625-32.

[26] Rocco B, Gregori A, Stener S, Santoro L, Bozzola A, Galli S, et al. Posterior reconstruction of the rhabdosphincter allows a rapid recovery of continence after transperitoneal videolaparoscopic radical prostatectomy. Eur Urol 2007; 51:996-1003.

[27] Wei JT, Dunn RL, Sandler HM, McLaughlin PW, Montie JE, Litwin MS, et al. Comprehensive comparison of health-related quality of life after contemporary therapies for localized prostate cancer. J Clin Oncol 2002; 20:557-66.

[28] Touijer K. Urinary continence after radical prostatectomy: "Beauty is in the eye of the beholder". Eur Urol 2007; 51:879-80.

[29] Stanford JL, Feng Z, Hamilton AS, Gilliland FD, Stephenson RA, Eley JW, et al. Urinary and sexual function after radical prostatectomy for clinically localized prostate cancer: The Prostate Cancer Outcomes Study. JAMA 2000; 283:354-60.

[30] McElveen TL, Waterman FM, Kim H, Dicker AP. Factors predicting for urinary incontinence after prostate brachytherapy. Int J Radiat Oncol Biol Phys 2004; 59:1395-404.

[31] Tseng TY, Kuebler HR, Cancel QV, Sun L, Springhart WP, Murphy BC, et al. Prospective health-related quality-of-life assessment in an initial cohort of patients undergoing robotic radical prostatectomy. Urology 2006; 68:1061-6.

[32] Hollenbeck BK, Dunn RL, Wei JT, Sandler HM, Sanda MG. Sexual health recovery after prostatectomy, external radiation, or brachytherapy for early stage prostate cancer. Curr Urol Rep 2004; 5:212-9.

[33] Berge V, Berg RE, Hoff JR, Wessel N, Diep LM, Karlsen SJ, et al. A prospective study of transition from laparoscopic to robot-assisted radical prostatectomy: Quality of life outcomes after 36-month follow-up. Urology 2013; 81:781-6.

[34] Si-Tu J, Lu MH, Li LY, Sun QP, Zhou XF, Qiu JG, et al. Prospective evaluation of pentafecta outcomes at 5 years after laparoscopic radical prostatectomy: Results of 170 patients at a single center. Neoplasma 2013; 60:309-14.

[35] Mishra S, Agrawal V, Khatri N, Sharma R, Kurien A, Ganpule A, et al. Laparoscopic radical prostatectomy: Oncological outcome analysis from a single-center Indian experience of 6 years. Indian J Urol 2012; 28:32-6.

[36] Montorsi F, Wilson TG, Rosen RC, Ahlering TE, Artibani W, Carroll PR, et al. Best practices in robot-assisted radical prostatectomy: Recommendations of the Pasadena Consensus Panel. Eur Urol 2012; 62:368-81. 